\title{
Treatment of group A streptococcal pharyngitis
}

$\mathrm{P}$ enicillin has long been the drug of choice for pharyngitis caused by group A streptococci, but its primacy has been challenged recently by reports of increasing incidence of treatment failures and the superiority of newer broad spectrum oral antimicrobial agents. The purpose of this note is to comment on recent information on antimicrobial therapy of streptococcal pharyngitis and to make recommendations concerning the optimal treatment of this common pediatric problem.

Group A streptococci have been identified as the cause of acute suppurative disease (particularly pharyngitis) and nonsuppurative, postinfectious complications (acute rheumatic fever and poststreptococcal glomerulonephritis) for many years. Penicillin has been shown to treat acute streptococcal pharyngitis effectively, shorten the clinical course of the disease, prevent suppurative complications, prevent transmission and prevent acute rheumatic fever. Despite the development of antimicrobial resistance among most common pediatric pathogens, group A streptococci remain uniformly susceptible to penicillin; penicillin is the drug of choice (recommended by the Canadian Paediatric Society, the American Academy of Pediatrics, the American Heart Association and the World Health Organization) for treatment of acute streptococcal pharyngitis. However, with the recognition of "treatment failures" among those who have received penicillin, concerns have been raised about whether this old stalwart should be replaced by newer antimicrobial agents, such as the extended spectrum cephalosporins or macrolides.

Evaluation of antimicrobial therapy for streptococcal pharyngitis is complicated by the high rate of pharyngeal carriage of group A streptococci among healthy children (up to $20 \%$ ). Because streptococci are often not eradicated by the treatment of carriers, this high background rate of carriage makes it extremely difficult to determine which children with

All material presented in Pediatric Infectious Disease Notes has been reviewed by Canadian Paediatric Society Board of Directors and members of the Canadian Paediatric Society Infectious Diseases and Immunization Committee

Correspondence: Infectious Diseases and Immunization Committee, Canadian Paediatric Society, 401 Smyth Road, Ottawa, Ontario K1H 8L1. Telephone 613-737-2728, fax 613-737-2794, e-mail journal@cps.ca pharyngitis are acutely infected with streptococci and which children who remain culture-positive following a course of antimicrobial therapy, represent true 'treatment failures' versus chronic carriers. Without measuring serological response (antibody to specific streptococcal antigens) or serotyping the cultured bacteria, one cannot differentiate true treatment failures from chronic carriers of group A streptococci. Unfortunately, seroconversion lags so far behind clinical illness that measuring antibody status is of no value during the acute illness. Also, antimicrobial therapy may reduce the serological response, further confounding the situation. Thus, the dilemma cannot be resolved at present, and one is unable to distinguish between the child who is acutely infected (who needs antimicrobial therapy) and the chronic carrier (who does not).

With the above caveats in mind, it has been widely accepted that antimicrobial therapy should be given to children with acute pharyngitis from whom group A streptococcus is identified in the throat (either by culture or antigen detection test). Landmark studies in the early 1950 s demonstrated that penicillin should be given orally for 10 days or as a single intramuscular dose of the benzathine formulation. Both regimens have been shown to eradicate group A streptococci from the pharynx of most children with acute streptococcal pharyngitis and to provide some symptomatic relief if given early enough in the course of the illness. Nonetheless, penicillin therapy often fails to eradicate the carriage state, a problem that leads to much confusion among practising clinicians and investigators and has prompted the search for superior alternative antimicrobial agents.

Recent reports have suggested two changes to the recommended regimen of 10 days of oral penicillin: the use of an alternative agent with broader antimicrobial spectrum, particularly against penicillinase-producing strains of oral bacteria; and the use of less than 10 days of therapy. To date there are insufficient data to support the routine implementation of either of these changes. The confounding effect of including chronic carriers has rendered the published studies (which compare penicillin with broader spectrum agents) extremely difficult to interpret because the broader spectrum agents may be superior to penicillin in eradicating the carrier state. The 
standard regimen of penicillin should not be abandoned unless newer, broader spectrum antimicrobials (with their attendant higher cost and enhanced risk of selecting resistant organisms) are shown to be superior at curing acute streptococcal pharyngitis; a careful review of the published data provides no compelling evidence that the newly proposed regimens satisfy this criterion. Whereas this note does not provide sufficient space to review the data clearly, the interested reader should see recently published reports that provide a balanced evaluation of the issue (see Recommended Readings).

In summary, the therapy of acute group A streptococcal pharyngitis remains a thorny, contentious issue, and physicians are seeking better therapeutic options to 10 days of oral penicillin. Nonetheless, penicillin has 'won its spurs' over the

ACKNOWLEDGMENT: Thanks to Dr Michael Gerber for his critical comments on this piece.

\section{RECOMMENDED READINGS}

Dajani A, Taubert K, Ferrieri P, Peter G, Shulman S. Treatment of acute streptococcal pharyngitis and prevention of rheumatic fever: A statement for health professionals. Pediatrics 1995;96:758-64.

Gerber MA. Treatment failures and carriers: perception or problems? Pediatr Infect Dis J 1994;13:576-9.

Markowitz M, Gerber MA, Kaplan EL. Treatment of streptococcal pharyngotonsillitis: Reports of penicillin's demise are premature. J Pediatr 1993:123:679-85.

Shulman ST. Evaluation of penicillins, cephalosporins and macrolides for therapy of streptococcal pharyngitis. Pediatrics 1996;97:955-9.

Sulman ST, Gerber MA, Tanz RR, Markowitz M. Streptococcal pharyngitis: The case for penicillin therapy. Pediatr Infect Dis ] 1994;13:1-7. past four decades and remains the drug of choice by virtue of its limited antimicrobial spectrum (with consistent activity against group A streptococcus), low cost, safety, capacity to prevent acute rheumatic fever and ability to shorten the course of streptococcal sore throat when initiated early in the illness. For a child with an acute sore throat from whom the group A streptococcus is identified (by culture or antigen detection), oral penicillin V ( $250 \mathrm{mg}$, two or three times per day) should be given for a full 10-day course. For adolescents and adults $500 \mathrm{mg}$ should be used.

Erythromycin is the drug of choice for patients allergic to penicillin. Penicillin should not be abandoned as the drug of choice of acute streptococcal pharyngitis until a study is conducted with sufficient power to demonstrate superiority of a different antimicrobial agent or regimen.

INFECTIOUS DISEASES AND IMMUNIZATION COMMITTEE Members: Drs Gilles Delage, Laboratoire de santé publique du Québec, Ste-Anne-de-Bellevue, Québec; François Boucher, Centre Hospitalier Universitaire de Québec, Québec, Québec; Joanne Embree, Winnipeg, Manitoba; Elizabeth Ford-Jones, The Hospital for Sick Children, Toronto, Ontario; David Speert (principal author), University of British Columbia, Vancouver, British Columbia; Ben Tan, Royal University Hopsital, University of Saskatchewan, Saskatoon, Saskatchewan. Liaisons: Drs Neal Halsey, Johns Hopkins University, Baltimore, Maryland (American Academy of Pediatrics); David Scheifele, Division of Infectious

Diseases, BC's Children's Hospital, Vancouver, British Columbia (Centre for Vaccine Evaluation); Susan Tamblyn, Perth District Health Unit, Stratford, Ontario (Public Health); John Waters, Alberta Health, Edmonton, Alberta (Epidemiology). Consultants: Drs Noni MacDonald, Division of Infectious Diseases, Children's Hospital of Eastern Ontario, Ottawa, Ontario; Victor Marchessault, Canadian Paediatric Society, Ottawa, Ontario 


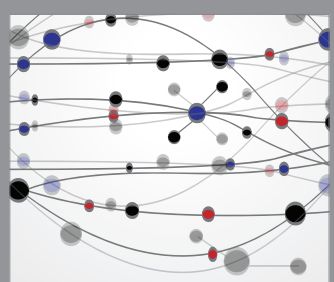

The Scientific World Journal
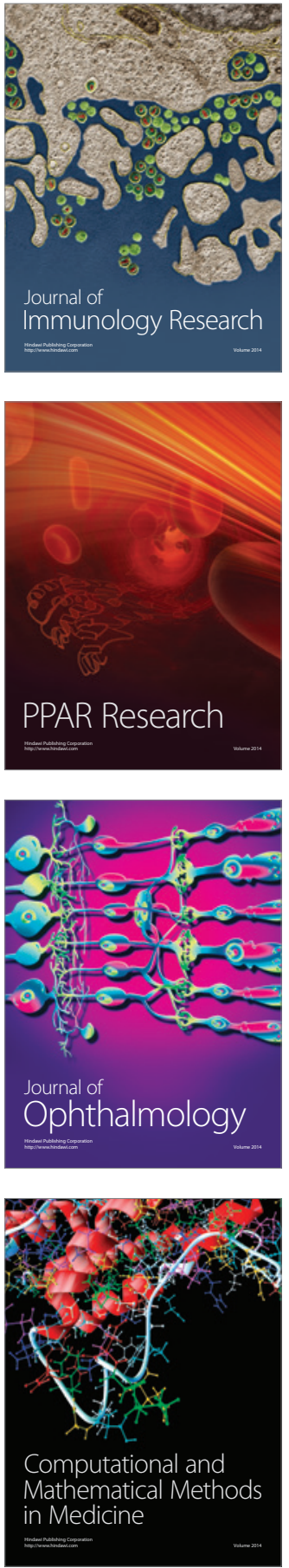

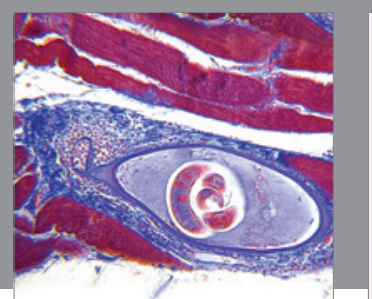

Gastroenterology Research and Practice

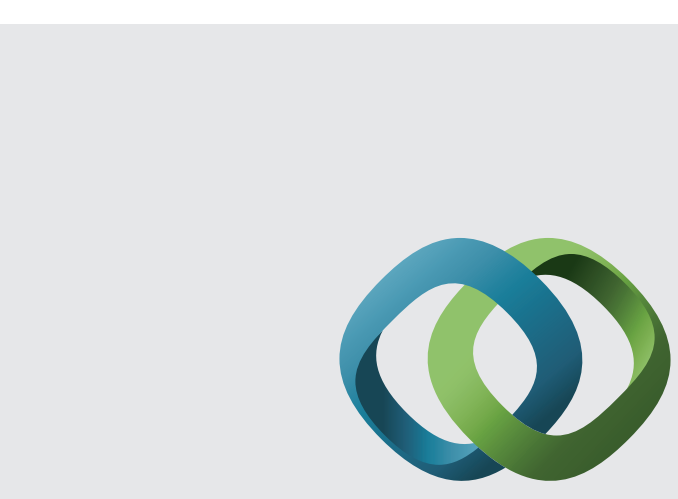

\section{Hindawi}

Submit your manuscripts at

http://www.hindawi.com
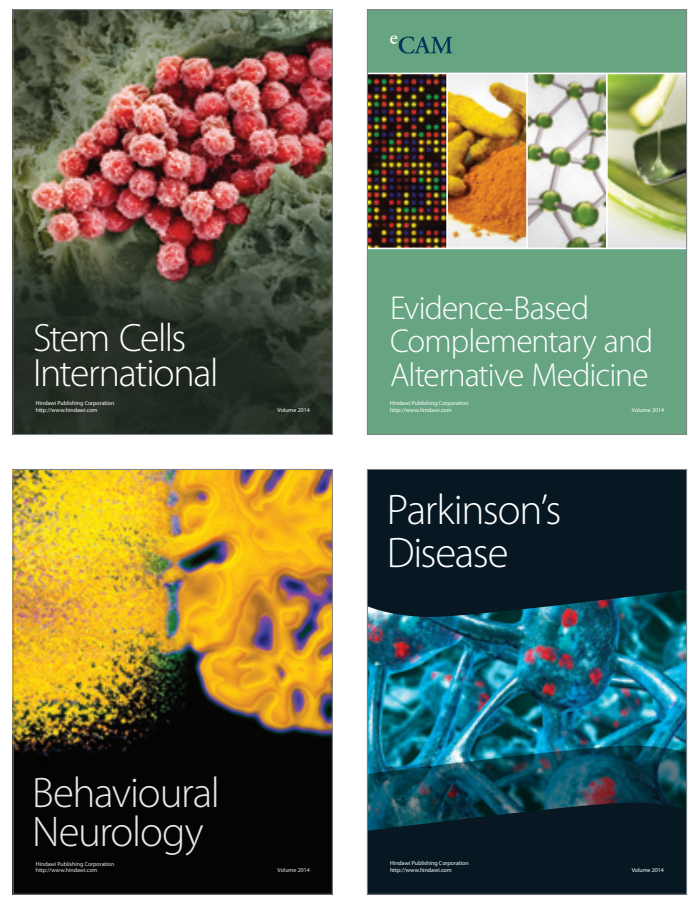
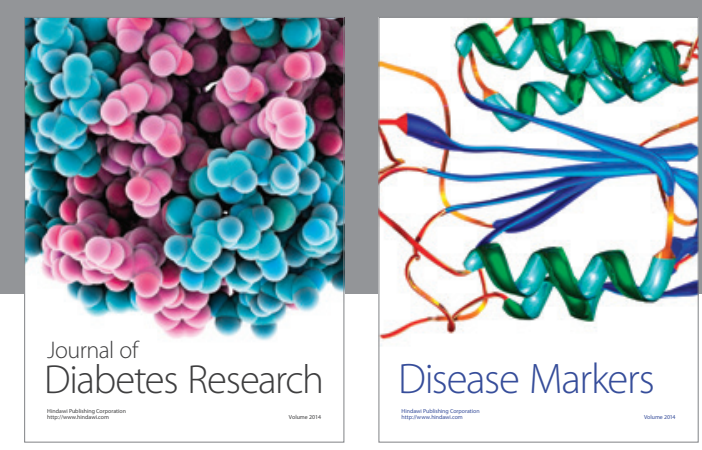

Disease Markers
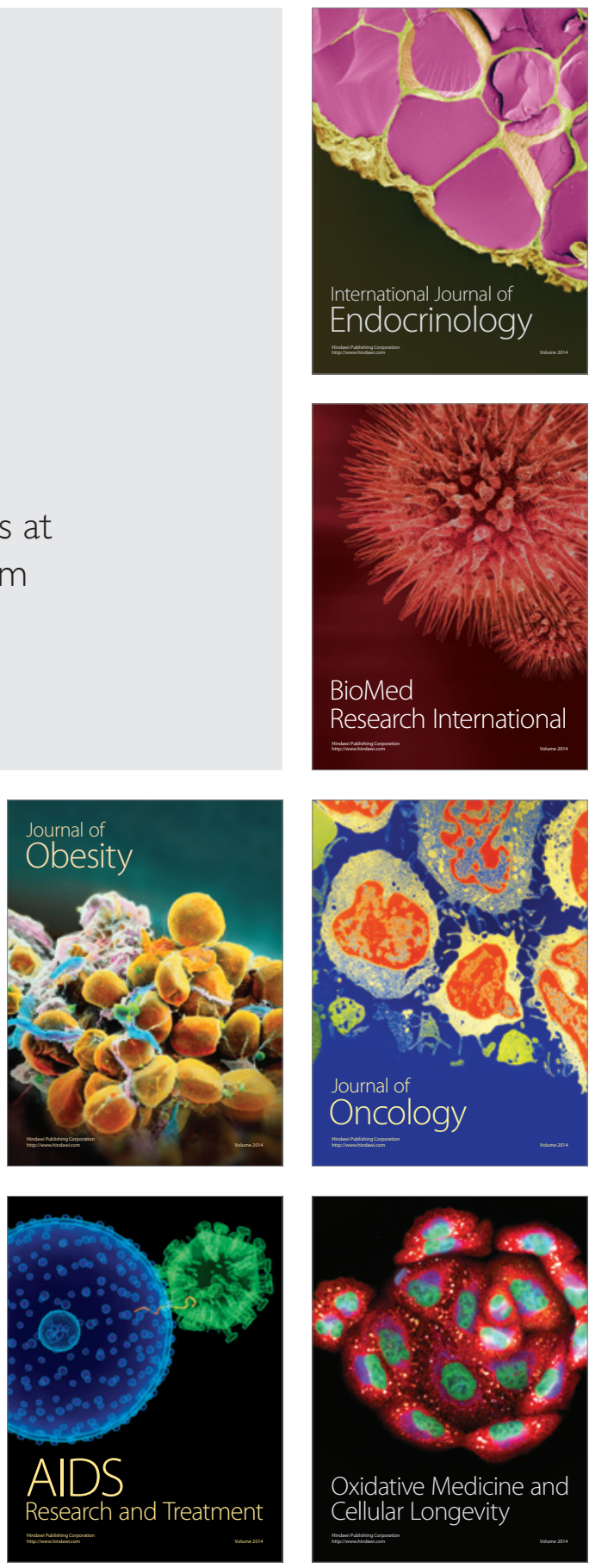